\title{
Induced hyperactivity in boar spermatozoa and its evaluation by computer-assisted sperm analysis
}

\author{
Harald Schmidt and Günter Kamp \\ Institute of Zoology, Johannes Gutenberg University, 55099 Mainz, Germany \\ Correspondence should be addressed to G Kamp; Email: kamp@uni-mainz.de
}

\begin{abstract}
Hyperactivity, a form of sperm motility characterized by vigorous flagellar movements, has been proposed as essential for fertilization in mammals. The objective of the present study was to establish a method for inducing hyperactivity in vitro in boar spermatozoa and to define threshold values to differentiate between hyperactive and non-hyperactive spermatozoa by computer-assisted sperm analysis (CASA) as a prerequisite for analyzing the energy metabolism during hyperactivity.

In TALP-HEPES medium, non-frozen boar spermatozoa were stimulated to hyperactivity by $50 \mu \mathrm{moll} \mathrm{I}^{-1} \mathrm{Ca}^{2+}$ within 15 min at $37^{\circ} \mathrm{C}$ if $5 \mathrm{\mu mol} \mathrm{I}^{-1}$ of the $\mathrm{Ca}^{2+}$ ionophore $\mathrm{A} 23187$ was present. If $25 \%$ seminal plasma was present, boar spermatozoa required higher $\mathrm{Ca}^{2+}$ concentrations (about $700 \mu \mathrm{moll}^{-1}$ ) for hyperactivity. Under both conditions, immobilization and headto-head agglutination were low so that hyperactive spermatozoa could be analyzed for at least 40 min. The transition from normal to hyperactive movement was characterized by an increase in flagellar beat angle from $49^{\circ} \pm 12^{\circ}$ to $200^{\circ} \pm 36^{\circ}$ $(n=32)$ and a decrease in flagellar curvature ratio from $0.89 \pm 0.04$ to $0.47 \pm 0.11(n=32)$. For quantification of hyperactive boar sperm, kinematic parameters of hyperactive and non-hyperactive spermatozoa were measured by CASA and statistically evaluated (receiver operating characteristic (ROC) curve analysis). The threshold values of the following four parameters were well suited for differentiating between hyperactive and non-hyperactive boar spermatozoa (ROC curve analysis: $>50 \%$ specificity at $\mathbf{1 0 0} \%$ sensitivity). Hyperactive boar spermatozoa showed mean lateral head displacement $>3.5 \mu \mathrm{m}$, curvilinear velocity $>97 \mu \mathrm{m} \mathrm{s}^{-1}$, linearity $<32 \%$ and wobble $<71 \%$. According to this multiparametric definition, induction of hyperactivity increased significantly $(P<0.0001)$ the fraction of hyperactive spermatozoa in semen samples from $5.1 \pm 4.3 \%$ $(n=13)$ to $48.3 \pm 6.6 \%(n=7)$ in the absence and to $44.2 \pm 7.6 \%(n=10)$ in the presence of $25 \%$ seminal plasma, while the overall percentage of motile spermatozoa did not change significantly.

Reproduction (2004) 128 171-179
\end{abstract}

\section{Introduction}

Hyperactive mammalian spermatozoa have been characterized by a vigorous and non-linear movement caused by an increased amplitude of flagellar beats (whiplash movement; for review see Yanagimachi 1994). This form of motility has been observed in sperm at the site and the time of fertilization (Suarez \& Ho 2003) and appears, therefore, to be essential for fertilization. It has been suggested that the mechanical thrust due to hyperactive motility is vital for spermatozoa to penetrate the zona pellucida of the oocyte (Yanagimachi 1969, Stauss et al. 1995, Bedford 1998). Additional functions, such as releasing spermatozoa that are attached to the oviductal epithelium (Suarez et al. 1991a, Demott \& Suarez 1992, Suarez 2002) and improving sperm movement through the mucus of the oviduct and cumulus matrix of the egg are also discussed (Suarez et al. 1991b, Suarez \& Dai 1992).
Sperm hyperactivity has been observed in vitro in various mammalian species especially if capacitation of spermatozoa was induced with capacitation media containing $\mathrm{Ca}^{2+}$ (for review see Yanagimachi 1994). Rapid hyperactivation was reported when $\mathrm{Ca}^{2+}$ influx was triggered by a $\mathrm{Ca}^{2+}$ ionophore (Suarez et al. 1987). The analysis of hyperactive boar spermatozoa is complicated because spermatozoa tend to agglutinate (head-to-head) in capacitation media containing $\mathrm{Ca}^{2+}$ (Harayama et al. 1998) and the $\mathrm{Ca}^{2+}$ ionophore A23187 tends to inactivate sperm (Suarez et al. 1987). Suarez and colleagues $(1987,1992)$ tried to overcome this problem by pulse stimulation in which they exposed sperm to the ionophore for only a short period and then absorbed the excessive ionophore by adding bovine serum albumin (BSA). In this paper we present a novel and simple method for inducing hyperactivity in boar spermatozoa by using low concentrations of 
$\mathrm{Ca}^{2+}$ and a constant concentration of the $\mathrm{Ca}^{2+}$ ionophore A23187. Both head-to-head agglutination as well as early inactivation are substantially prevented, and hyperactivity of boar spermatozoa can be studied for $40 \mathrm{~min}$ or more.

Computer-assisted sperm analysis (CASA) is a powerful tool for the objective assessment of sperm motility and is hence frequently used for evaluating the quality of semen from man and animals (Mortimer 1997, 2000, Verstegen et al. 2002). However, the quantitative evaluation of semen by CASA is based on kinematic parameters of individual spermatozoa that are reconstructed merely from the movements of their heads, because CASA instrumentation does not usually allow the direct analysis of flagellar movements that generate sperm hyperactivity (Mortimer 1997). Hence, to detect hyperactive spermatozoa by CASA requires kinematic parameters of sperm head movements that are correlated with characteristic changes in flagellar movements. For the accurate assessment of hyperactive spermatozoa by CASA, the set of parameters must be defined for a particular species, taking into account the image sampling frequency (Mortimer \& Maxwell 1999).

Threshold kinematic parameters to differentiate between hyperactive and non-hyperactive spermatozoa have been reported for mouse (Neill \& Olds-Clarke 1987), man (for review and citations see Mortimer 1997), ram (Mortimer \& Maxwell 1999) and rat (Cancel et al. 2000). Hyperactivation in boar spermatoza has been mentioned several times (Saxena et al. 1986, Blottner et al. 1989, Hamano et al. 1989), but an analysis of movement patterns in ejaculated boar spermatozoa and in spermatozoa flushed from the oviducts of pigs has only been reported by Suarez et al. (1992). A detailed investigation of hyperactivated boar spermatozoa using CASA has not yet been reported. Therefore, we compared the two characteristic parameters of sperm flagellar movement, the flagellar beat angle (FBA) and the flagellar curvature ratio (FCR) with kinematic parameters of CASA in order to define objective kinematic thresholds that can be used to identify hyperactive boar spermatozoa. This identification and quantification of hyperactive spermatozoa is necessary for analyzing the energy metabolism of hyperactivity now in progress. We propose that hyperactivity depends on glycolytic ATP production in the principal piece of the flagellum (Westhoff \& Kamp 1997, Bone et al. 2000).

\section{Materials and Methods}

\section{Chemicals}

BTS (Beltsville thawing solution) was from Minitüb (Tiefenbach, Germany) and gentamicin was from Denk-Chemie (München). All other chemicals were from Sigma-Aldrich (Taufkirchen, Germany) or Merck (Darmstadt, Germany).

\section{Collection and storage of semen}

Semen, collected from fertile boars kept for commercial artificial insemination, was provided by GFS (Genossenschaft zur Förderung der Schweinehaltung, Ascheberg, Germany). The semen was diluted with the same volume of BTS and stored at $17^{\circ} \mathrm{C}$ until use (non-cryopreserved). All experiments were performed with semen stored liquid for only 1 day. The final concentration of boar spermatozoa was about $20 \times 10^{6}$ per $\mathrm{ml}$ in all experiments.

\section{Media and reagents}

BTS containing $250 \mathrm{mg}_{\text {gentamicin }}{ }^{-1}$ was used for diluting boar semen. TALP-HEPES medium (pH 7.4) (Bavister \& Yanagimachi 1977) comprised $6 \mathrm{mmoll}^{-1}$ glucose, $113 \mathrm{mmol} \mathrm{I}^{-1} \mathrm{NaCl}, 2.7 \mathrm{mmol} \mathrm{I}^{-1} \mathrm{KCl}, 0.5 \mathrm{mmol} \mathrm{I}^{-1} \mathrm{MgCl}_{2}$, $0.4 \mathrm{mmolI}^{-1} \mathrm{NaH}_{2} \mathrm{PO}_{4}, 20 \mathrm{mmolI}^{-1} \mathrm{NaHCO}_{3}, 1 \mathrm{mmoll}^{-1}$ pyruvate, $20 \mathrm{mmolI}^{-1}$ lactate, $20 \mathrm{mmol} \mathrm{I}^{-1}$ HEPES and $0.6 \%$ BSA. The $\mathrm{Ca}^{2+}$ ionophore A23187 was added from a stock solution ( $1 \mathrm{mmol} \mathrm{I}^{-1}$ dissolved in DMSO). The final concentration of the $\mathrm{Ca}^{2+}$ ionophore was $5 \mu \mathrm{moll}^{-1}$ in all experiments. Equivalent amounts of DMSO were added to controls.

Seminal plasma is the supernatant of boar semen after centrifugation at $10000 \mathrm{~g}$ for $10 \mathrm{~min}$ at $4{ }^{\circ} \mathrm{C}$.

\section{Hyperactivation of spermatozoa}

Hyperactivation was induced either in the absence or in the presence of seminal plasma. For hyperactivation in the absence of seminal plasma, prediluted semen stored at $17^{\circ} \mathrm{C}$ was centrifuged at $500 \mathrm{~g}$ for $2 \mathrm{~min}$, the supernatant discarded and the sedimented spermatozoa were resuspended in TALP-HEPES. After addition of $50 \mu \mathrm{mol} \mathrm{I}^{-1}$ $\mathrm{CaCl}_{2}$ and $5 \mu \mathrm{moll}^{-1}$ of the $\mathrm{Ca}^{2+}$ ionophore $\mathrm{A} 23187$, the suspension was incubated at $37^{\circ} \mathrm{C}$ for $15 \mathrm{~min}$ and analyzed by CASA. For hyperactivation in the presence of seminal plasma, the sperm sediment was resuspended in a medium comprising 50\% TALP-HEPES, 25\% BTS and $25 \%$ pooled undiluted seminal plasma that had been collected from 12 boars. $\mathrm{CaCl}_{2}\left(700 \mu \mathrm{moll}{ }^{-1}\right)$ was added and samples were analyzed $15 \mathrm{~min}$ after the addition of $5 \mu \mathrm{mol} \mathrm{I}{ }^{-1} \mathrm{Ca}^{2+}$ ionophore (incubated at $37^{\circ} \mathrm{C}$ ).

\section{Analysis of sperm motility}

Aliquots of $2.2 \mu \mathrm{l}$ sperm suspension were pipetted into disposable counting chambers (standard count 4-chamber 20 micron slides, Leja, Nieuw Vennep, the Netherlands) that had been prewarmed to $37^{\circ} \mathrm{C}$ on a heated microscope stage. Motility was observed using a Leica inverted microscope (Leitz DM IRB, Leica, 64625 Bensheim) with a 20-times magnification objective for negative phase contrast. Spermatozoa were monitored with an analogous camera (Kappa CF 8/4, 37130 Gleichen) using either a 1:1 or 1:0.5 C-mount (Leica, 64625 Bensheim) and 50 pictures per s $(50 \mathrm{~Hz})$ were recorded via a video cassette 
recorder (Panasonic NV-HS800). A TV-screen (Sony KVM1430D) was used for monitoring sperm motility.

\section{Analysis of flagellar movement}

The method of Mortimer and Maxwell (1999) for analyzing ram spermatozoa was slightly modified. For flagellar movement analysis, videotapes were replayed and projected (Panasonic PT-L795E LCD projector) on white paper attached to a magnetic rotatable table. Flagellar bends were directly copied on to the paper with respect to the position of the sperm head. The angle between the tangents of the outer flagellar waves was determined manually resulting in the flagellar beat angle (FBA, cf. Fig. 1). Additionally, flagellar curvature ratio (FCR) was measured manually from the flagellar movements drawn for FBA analysis. FCR is the straight line distance from the neck (junction between the head and the mid piece) to the first inflection point of the tail divided by the distance on the curve between the same points (cf. Fig. 1; Suarez 1988). The inflection point was estimated as the intersection of the flagellar wave with a parallel straight line amidst two parallel tangents drawn on the outer flagellar waves.

\section{Computer-assisted sperm analysis}

Videotapes were digitalized by a frame-grabber (Vidisys, 82054 Sauerlach, Germany) and analyzed by a cell motion analyzer program from Strömberg-Mika (SM-CMA, Version 2.0, GAMBIT Technologies AG, 6330 Cham, Switzerland). Spermatozoa were identified as immotile by tail detection and average path velocity (VAP) below $10 \mu \mathrm{m} \mathrm{s}^{-1}$. Spermatozoa with a VAP between 10 and
$20 \mu \mathrm{m} \mathrm{s}^{-1}$ were classified as local motile or non-progressive motile; those with VAP exceeding $20 \mu \mathrm{m} \mathrm{s}^{-1}$ as motile (progressive motile).

For each trajectory, 50-60 images collected at $50 \mathrm{~Hz}$ over 1 to $1.2 \mathrm{~s}$ were analyzed. The following kinematic parameters (for definitions see Mortimer 1997) were measured for each trajectory: curvilinear velocity $(\mathrm{VCL}$, $\mu \mathrm{m} \mathrm{s}^{-1}$ ), straight line velocity (VSL, $\mu \mathrm{m} \mathrm{s}^{-1}$ ), average path velocity $\left(\mathrm{VAP}, \mu \mathrm{m} \mathrm{s}^{-1}\right)$, mean lateral head displacement $\left(\mathrm{LHD}_{\text {mean }}, \mu \mathrm{m}\right)$ and beat cross frequency $(\mathrm{BCF}, \mathrm{Hz})$. From the kinematic parameters, linearity $(\mathrm{LIN}=\mathrm{VSL} \times 100$ / $\mathrm{VCL})$, straightness $(\mathrm{STR}=\mathrm{VSL} \times 100 / \mathrm{VAP})$ and wobble $(\mathrm{WOB}=\mathrm{VAP} \times 100 / \mathrm{VCL})$ were calculated.

\section{Statistics}

To tell non-hyperactive from hyperactive spermatozoa, threshold values with the sensitivity and specificity at highest accuracy were calculated for different parameters using ROC (receiver operating characteristic) curve analysis and the computer program MedCalc for Windows (Medcalc, Mariakerke, Belgium). One hundred per cent sensitivity corresponds to the probability of detecting all actually hyperactive spermatozoa. Similarly, $100 \%$ specificity corresponds to the probability of detecting all non-hyperactive spermatozoa. Values are given as means \pm S.D., and significant differences between means were calculated with Microsoft Excel. According to the result of a variance ratio analysis (F-test, $P<0.01$ ), an unpaired $t$-test for either homoscedastic or heteroscedastic variances was performed $(P<0.0001)$.
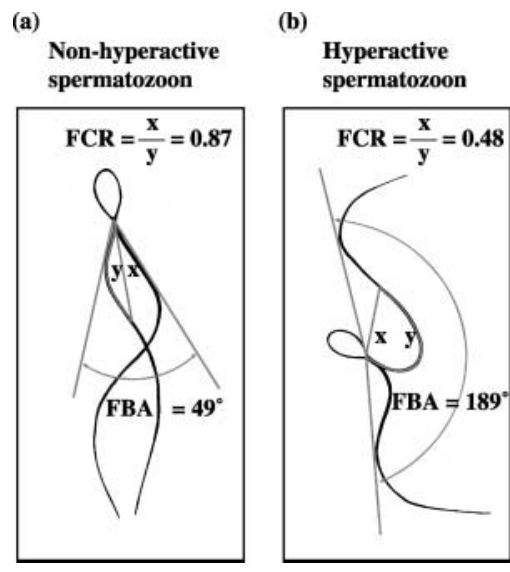

(c)

Flagellar beat angle (FBA)

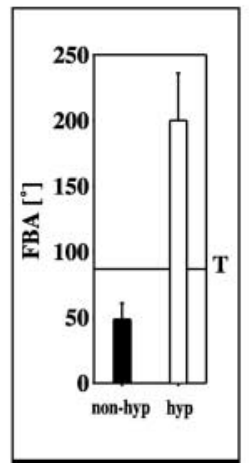

(d)

Flagellar curvation ratio (FCR)

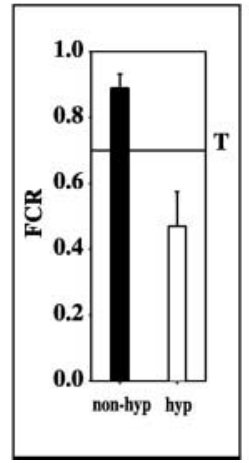

Figure 1 Flagellar beat angle (FBA) and flagellar curvature ratio (FCR) of non-hyperactive and hyperactive boar spermatozoa. Spermatozoa with typical flagellar movements of non-hyperactivity and hyperactivity were selected on videotapes of semen diluted with BTS or incubated with TALP-HEPES in the presence of $25 \%$ seminal plasma, $700 \mu \mathrm{mol}{ }^{-1} \mathrm{Ca}^{2+}$ and $5 \mu \mathrm{moll}{ }^{-1} \mathrm{Ca}^{2+}$ ionophore A23187 for $15 \mathrm{~min}$ at $37^{\circ} \mathrm{C}$. FBA and FCR were determined from 32 non-hyperactive or 32 hyperactive spermatozoa (see Materials and Methods). FBA is the angle between the tangents of the outer flagellar waves and FRC is the quotient of straight $(x)$ and curve $(y)$ line distances both measured from the head junction to the first inflection point of the flagellum as exemplified in (a) and (b) for a non-hyperactive and a hyperactive spermatozoon respectively. FBA and FRC values \pm S.D. of 32 non-hyperactive (non-hyp) and 32 hyperactive (hyp) spermatozoa are given in (c) and (d). The lines (T) show the threshold values separating hyperactive from non-hyperactive spermatozoa. FBA and FRC of hyperactive and non-hyperactive spermatozoa were significantly different $(t$-test, $P<0.0001)$. 


\section{Results}

\section{Motility of boar spermatozoa in prediluted semen}

Boar semen diluted with the same volume of BTS usually contained $80 \pm 10 \%$ motile spermatozoa $\left(37^{\circ} \mathrm{C}\right)$. Semen samples could be stored for up to 2 days at $17^{\circ} \mathrm{C}$ without significant loss of sperm motility. In all experiments 1 -dayold fresh semen was analyzed.

\section{Analysis of flagellar movement in hyperactive boar spermatozoa}

Incubation of boar spermatozoa at $37^{\circ} \mathrm{C}$ with TALP-HEPES in the presence of $25 \%$ seminal plasma, $700 \mu \mathrm{mol} \mathrm{I}^{-1}$ $\mathrm{Ca}^{2+}$ and $5 \mathrm{mmoll}^{-1} \mathrm{Ca}^{2+}$ ionophore resulted, after $15 \mathrm{~min}$, in an almost synchronous change to vigorous motility with whiplash-like flagellar movements as is typical of hyperactivity (Fig. 1). The FBA increased from $49^{\circ} \pm 12^{\circ}$ in the control group to $200^{\circ} \pm 36^{\circ}$ in the hyperactive spermatozoa while the FCR decreased from $0.89 \pm 0.04$ in controls to $0.47 \pm 0.11$ in the hyperactive group. Both effects were highly significant $(P<0.0001, n=32)$. Hence, according to the definition of hyperactive spermatozoa from several species (Suarez \& Osman 1987, Suarez 1988, Suarez et al. 1992, Mortimer et al. 1997, Mortimer \& Maxwell 1999), the treatment stimulated spermatozoa to hyperactivity. The threshold values obtained from the ROC curve analysis to differentiate between non-hyperactive and hyperactive spermatozoa were $87^{\circ}$ for FBA and 0.7 for FCR at $100 \%$ specificity and $100 \%$ sensitivity.

\section{Kinematic parameters of CASA for hyperactivity}

For computer-assisted quantification of hyperactive boar spermatozoa the kinematic parameters of hyperactive and non-hyperactive boar spermatozoa identified by FBA and FCR (Fig. 1) were determined by CASA (Table 1). VCL and $\mathrm{LHD}_{\text {mean }}$ were significantly increased $(P<0.0001)$ while LIN, STR, WOB and BCF were significantly decreased $(P<0.0001)$ upon hyperactivation. The most pronounced

Table 1 Kinematic parameters for hyperactive and non-hyperactive boar spermatozoa. Spermatozoa selected for analysis of flagellar movements (Fig. 1) were subsequently evaluated by CASA. A total number of 32 sperms were analysed for each group. Values are given as means \pm S.D. $(n=32)$.

\begin{tabular}{|c|c|c|}
\hline Parameter $^{+}$ & Non-hyperactive sperm & Hyperactive sperm \\
\hline $\operatorname{VCL}\left(\mu \mathrm{m} \mathrm{s}^{-1}\right)$ & $84.2 \pm 24.0$ & $151.6 \pm 33.5 *$ \\
\hline $\operatorname{VSL}\left(\mu \mathrm{m} \mathrm{s}^{-1}\right)$ & $35.5 \pm 26.6$ & $16.8 \pm 9.1$ \\
\hline $\operatorname{VAP}\left(\mu \mathrm{m} \mathrm{s}^{-1}\right)$ & $71.1 \pm 21.5$ & $88.1 \pm 18.6$ \\
\hline $\mathrm{LHD}_{\text {mean }}(\mu \mathrm{m})$ & $1.3 \pm 0.5$ & $8.0 \pm 1.8 *$ \\
\hline LIN $(\%)$ & $41.5 \pm 23.5$ & $11.8 \pm 7.3 *$ \\
\hline STR (\%) & $48.2 \pm 26.0$ & $20.8 \pm 13.6 *$ \\
\hline WOB $(\%)$ & $84.6 \pm 7.2$ & $58.6 \pm 6.3 *$ \\
\hline $\mathrm{BCF}(\mathrm{Hz})$ & $19.2 \pm 4.2$ & $8.0 \pm 2.1 *$ \\
\hline
\end{tabular}

${ }^{\dagger}$ For definition see Mortimer (1997).

$* P<0.0001$ compared with non-hyperactive sperm. change was found in LHD $_{\text {mean }}$ which increased about sixfold with hyperactivation from 1.3 to $8.0 \mu \mathrm{m}$.

The ROC curve analysis confirms $\mathrm{LHD}_{\text {mean }}$ as a very distinct parameter for differentiating hyperactive from non-hyperactive spermatozoa (Table 2 ). The sensitivity and specificity are $100 \%$ at the threshold value $\left(\mathrm{LHD}_{\text {mean }}\right.$ $>3.5 \mu \mathrm{m}$ for hyperactive spermatozoa). Additionally, WOB, VCL and LIN all showed approximately 100\% sensitivity with high specificity $(>50 \%)$. For a multiparametric definition of hyperactive boar spermatozoa, the following threshold values were used in combination: VCL $>97 \mu \mathrm{ms}^{-1}, \mathrm{LHD}_{\text {mean }}>3.5 \mu \mathrm{m}, \mathrm{LIN}<32 \%$ and WOB $<71 \%$.

The percentage of hyperactive sperm detected in boar samples before and after induction of hyperactivity was lower (not significant at $P<0.01$ ) in this multiparametric analysis than in a monoparametric analysis using $\mathrm{LHD}_{\text {mean }}$ as the only indicator (Table 3). This was due to by more false positive identifications by the monoparametric compared with the multiparametric analysis.

\section{Calcium concentration and hyperactivation}

When seminal plasma was absent (as in TALP-HEPES medium), the addition of 40 to $60 \mu \mathrm{molI}^{-1} \mathrm{Ca}^{2+}$ was necessary for maximum hyperactivation and for maintaining high motility (Fig. 2a). Below $30 \mu \mathrm{moll}^{-1}$ and above $60 \mu \mathrm{moll}^{-1} \mathrm{Ca}^{2+}$ loss of motility and hyperactivity was observed in the presence of the calcium ionophore.

In the presence of $25 \%$ seminal plasma, 10-fold higher concentrations of $\mathrm{Ca}^{2+}$ proved necessary for hyperactivation (Fig. 2b). Calcium concentrations lower than $500 \mu \mathrm{mol} \mathrm{I}^{-1}$ did not induce hyperactivation and concentrations higher than $900 \mu \mathrm{moll}^{-1}$ resulted in immotility.

\section{Time course of induced hyperactivity}

Spermatozoa were incubated at $37^{\circ} \mathrm{C}$ in TALP-HEPES media containing 50 or $700 \mu \mathrm{moll}^{-1} \mathrm{Ca}^{2+}$ in the presence or absence of $25 \%$ seminal plasma respectively. About $15 \mathrm{~min}$ after the addition of the $\mathrm{Ca}^{2+}$ ionophore A23187, approximately $60 \%$ of spermatozoa had switched from non-hyperactive to hyperactive motility and maintained hyperactivity for at least $40 \mathrm{~min}$ (Fig. 3). Thereafter, the percentage of motile and hyperactive motile spermatozoa slowly decreased and sometimes head-tohead agglutination was observed in the absence of seminal plasma, while tail-to-tail agglutination was found in the presence of $25 \%$ seminal plasma.

\section{Hyperactivity of boar sperm samples}

Hyperactivity was induced in sperm samples from 13 fertile boars (Table 4). The percentage of hyperactive spermatozoa was significantly $(P<0.0001)$ increased after induction of hyperactivity. In the absence of seminal plasma $63 \%$ of motile spermatozoa were hyperactive and in the presence of seminal plasma 58\% were hyperactive. 
Table 2 ROC analysis of hyperactivated boar spermatozoa. Thirty-two hyperactivated and 32 non-hyperactivated spermatozoa were analyzed. Threshold values were calculated from data of 5 independent analyses for each spermatozoon. Sensitivity is the probability that a test will be positive when hyperactive sperm are present. Specificity is the probability that a test result will be negative when hyperactive sperm are not present. The threshold values (col. 1) are given for the highest accuracy together with the respective sensitivity (col. 2) and specificity (col. 3). Additionally, the threshold values (col. 4) and the specificity (col. 5) were determined for $100 \%$ sensitivity. At $100 \%$ sensitivity, the threshold values of $\mathrm{VCL}(>97), \mathrm{LHD}(>3.5)$, LIN $(<32)$ and WOB $(<71)$ show more than $50 \%$ specificity and were selected for a multiparametric definition of hyperactive boar spermatozoa.

\begin{tabular}{|c|c|c|c|c|c|}
\hline \multirow[b]{2}{*}{ Parameter } & \multicolumn{3}{|c|}{ Values for highest accuracy } & \multicolumn{2}{|c|}{$100 \%$ Sensitivity } \\
\hline & Threshold (col. 1) & Sensitivity (col. 2) & Specificity (col. 3) & Threshold (col. 4) & Specificity (col. 5) \\
\hline $\operatorname{VCL}\left(\mu \mathrm{ms}^{-1}\right)$ & $>104.8$ & 98.1 & 83.7 & $>97.4$ & 80.6 \\
\hline $\operatorname{VSL}\left(\mu \mathrm{m} \mathrm{s}^{-1}\right)$ & $\leq 20.3$ & 68.1 & 68.1 & $\leq 51.1$ & 17.5 \\
\hline $\operatorname{VAP}\left(\mu \mathrm{m} \mathrm{s}^{-1}\right)$ & $>73.7$ & 75.0 & 70.6 & $>48.0$ & 6.9 \\
\hline $\mathrm{LHD}_{\text {mean }}(\mu \mathrm{m})$ & $>3.5$ & 100 & 100 & $>3.5$ & 100 \\
\hline $\operatorname{LIN}(\%)$ & $\leq 31.7$ & 100 & 59.4 & $\leq 31.7$ & 59.4 \\
\hline WOB (\%) & $<71.3$ & 100 & 94.4 & $<71.3$ & 94.4 \\
\hline STR (\%) & $<48.4$ & 93.7 & 49.4 & $<71.33$ & 26.2 \\
\hline $\mathrm{BCF}(\mathrm{Hz})$ & $\leq 10.0$ & 92.5 & 92.5 & $\leq 23.33$ & 1.9 \\
\hline
\end{tabular}

Definitions for CASA parameters are given in Mortimer (1997).

\section{Discussion}

Hyperactive motility of mammalian spermatozoa is regarded as important for penetrating the zona pellucida of oocytes (for review see Yanagimachi 1994) and has therefore been proposed for assessment of semen quality, particularly in men (Burkman 1990, Mortimer 1997, Kay \& Robertson 1998). Freshly ejaculated semen usually contains only a minor fraction of hyperactive spermatozoa. Rather, hyperactivity develops during capacitation either in vivo or in vitro. We have found $5.1 \pm 4.3 \%$ $(n=13)$ spermatozoa to be hyperactive in 13 fertile boar semen 1 day after ejaculation. Hyperactivity, however, could be induced in up to $60 \%$ of motile spermatozoa by the methods presented in this paper. These methods enable the study of the energy metabolism of hyperactive spermatozoa (see below).

\section{Hyperactivation and $\mathrm{Ca}^{2+}$}

Hyperactivity is apparently induced during sperm capacitation in the female genital tract, but neither the extracellular trigger nor the pathway of signal transduction are known. As an intracellular signal, $\mathrm{Ca}^{2+}$ is involved in the control of sperm hyperactivity. Demembranated bull spermatozoa became motile and increasingly hyperactive at very low $\mathrm{Ca}^{2+}$ concentrations $\left(>0.05 \mu \mathrm{mol}{ }^{-1}\right)$. About $70 \%-80 \%$ of sperm were hyperactive between 0.4 and $100 \mathrm{mmoll}^{-1} \mathrm{Ca}^{2+}$, but at $1 \mathrm{mmoll}^{-1} \mathrm{Ca}^{2+}$ both the non-hyperactive and hyperactive motility decreased (Ho et al. 2002).

Hyperactivity has also been induced in intact spermatozoa of several mammalian species by capacitation media containing $\mathrm{Ca}^{2+}$ in the $\mathrm{mM}$ range (for review see Yanagimachi 1994). Capacitation is accompanied by changes in membrane structure thus increasing $\mathrm{Ca}^{2+}$ influx which triggers hyperactivity and, finally, the acrosome reaction. As a consequence of changes in membrane structure spermatozoa tend to aggregate headto-head, especially in boars (Harayama et al. 1998). The motility of aggregated spermatozoa, however, cannot be analyzed by CASA and therefore capacitation with $\mathrm{mM}$ $\mathrm{Ca}^{2+}$ hampers the analysis of hyperactivity, at least in boar spermatozoa.

In intact mouse and boar spermatozoa, transient hyperactivity was also observed if $\mathrm{Ca}^{2+}$ influx was brought about by the $\mathrm{Ca}^{2+}$ ionophore A23187 (Suarez et al. 1987, 1992). The degree of hyperactivation seems to be dependent on various factors, particularly on the concentrations

Table 3 Percentage of hyperactive spermatozoa in boar semen samples determined by a monoparametric and multiparametric CASA analysis. Semen from 5 boars was evaluated by CASA. Sperm were either untreated (non-hyperactivated) or hyperactivated in the presence of $25 \%$ seminal plasma. Motility of sperm was recorded on video tapes and sperm were classified with CASA using the monoparametric (LHD $>3.5 \mu \mathrm{m}$ ) or the multiparametric definition ( $\mathrm{VCL}>97 \mu \mathrm{m} / \mathrm{s}$, LHD $>3.5 \mu \mathrm{m}, \mathrm{LIN}<32 \%$ and WOB $<71 \%$ ). Values are given in percent means \pm S.D. $100 \%$ represents total number of spermatozoa (motile, immotile and locally motile sperm).

\begin{tabular}{|c|c|c|c|c|}
\hline & \multicolumn{2}{|c|}{ Non-hyperactivated } & \multicolumn{2}{|c|}{ Hyperactivated } \\
\hline & Monoparametric & Multiparametric & Monoparametric & Multiparametric \\
\hline Motile & $78.6 \pm 5.6$ & $79.2 \pm 5.8$ & $80.7 \pm 6.5$ & $80.2 \pm 6.7$ \\
\hline Hyperactive motile & $8.2 \pm 7.7$ & $5.9 \pm 6.6$ & $66.9 \pm 10.3$ & $46.6 \pm 9.9$ \\
\hline
\end{tabular}

The monoparametric definition resulted in a non-significant increase at $P<0.01$ of sperm identified as hyperactive, especially when hyperactivated samples were analyzed. 
(a)

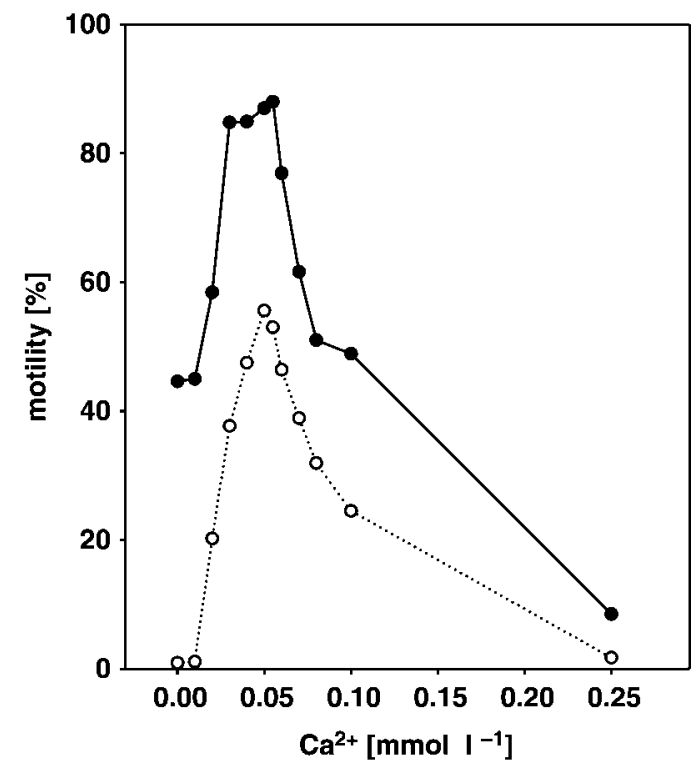

(b)

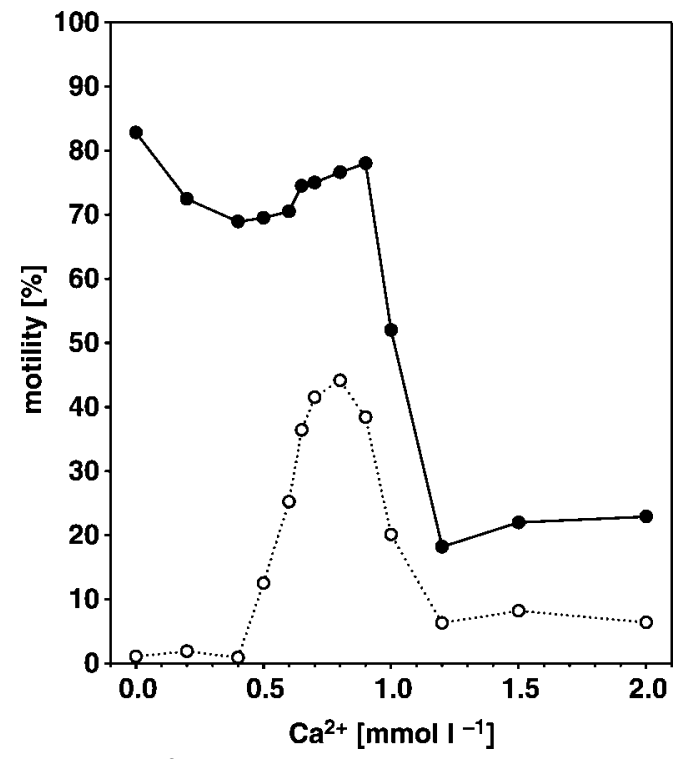

Figure 2 Effect of $\mathrm{Ca}^{2+}$ concentration on hyperactivation of boar spermatozoa in (a) the absence and (b) the presence of seminal plasma. (a) Prediluted boar semen was centrifuged ( $500 \mathrm{~g}, 2 \mathrm{~min}$ ), and the spermatozoa were resuspended in TALP-HEPES yielding a final concentration of about $20 \times 10^{6}$ spermatozoa per $\mathrm{ml}$. Hyperactivity was induced by adding $5 \mu \mathrm{mol} \mathrm{I}{ }^{-1} \mathrm{Ca}^{2+}$ ionophore at various $\mathrm{Ca}^{2+}$ concentrations. After $15 \mathrm{~min}$ of incubation $\left(37^{\circ} \mathrm{C}\right)$, the percentage of motile (hyperactive plus non-hyperactive, $\mathbf{0}$ ) and hyperactive (O) spermatozoa was determined by multiparametric CASA. One hundred percent refers to the total number of spermatozoa analyzed (immotile, local motile and motile spermatozoa). The percentage of hyperactive spermatozoa was highest at $0.05 \mathrm{mmol}^{-1} \mathrm{Ca}^{2+}$. (b) Prediluted boar semen was centrifuged ( $500 \mathrm{~g}, 2 \mathrm{~min}$ ) and the spermatozoa were resuspended in TALPHEPES $(50 \% \mathrm{v} / \mathrm{v})$, BTS $(25 \% \mathrm{v} / \mathrm{v})$ and seminal plasma $(25 \% \mathrm{v} / \mathrm{v})$ yielding a final concentration of about $20 \times 10^{6}$ spermatozoa per $\mathrm{ml}$. Hyperactivation was induced and analyzed as in (a). After $15 \mathrm{~min}$ incubation $\left(37^{\circ} \mathrm{C}\right)$, the percentage of motile $(\bullet)$ and hyperactive $(O)$ spermatozoa was determined as in (a). The highest percentage of hyperactive spermatozoa was seen at $0.8 \mathrm{mmoll}^{-1} \mathrm{Ca}^{2+}$. of $\mathrm{Ca}^{2+}$ and of the ionophore. Mouse spermatozoa were made hyperactive for several minutes by incubating them with $1 \mathrm{mmoll}^{-1} \mathrm{Ca}^{2+}$ and $1 \mu \mathrm{molI}^{-1}$ ionophore and attenuating the effect of the ionophore by albumin (Suarez et al. 1987). A similar procedure as in mice was used to hyperactivate boar spermatozoa (Suarez et al. 1992).

Unlike the approach above, we have varied the $\mathrm{Ca}^{2+}$ concentration and kept the concentration of the ionophore constant. Under these conditions a specific range of $\mathrm{Ca}^{2+}$ concentrations induces hyperactivity in boar spermatozoa. Using this method, boar spermatozoa remain hyperactive for at least $40 \mathrm{~min}$ which is long enough for studying the energy metabolism of hyperactive boar spermatozoa (see below). Sperm freed from seminal plasma by mild centrifugation $(500 \mathrm{~g}, 2 \mathrm{~min})$ and incubated in TALP-HEPES medium became hyperactive in the narrow range of 40 to $70 \mu \mathrm{mol} \mathrm{Ca}^{2+} \mathrm{I}^{-1}$. High $\mathrm{Ca}^{2+}$ concentrations $\left(>100 \mu \mathrm{moll}{ }^{-1}\right)$ resulted in a nearly complete loss of sperm motility and low $\mathrm{Ca}^{2+}$ concentrations $\left(<20 \mu \mathrm{moll}^{-1}\right)$ prevented hyperactivity and reduced sperm motility. These responses of intact boar spermatozoa to $\mathrm{Ca}^{2+}$ in the presence of the ionophore are strikingly similar to those of demembranated bull spermatozoa (Ho et al. 2002). Hence, the use of the ionophore and a precisely adjusted $\mathrm{Ca}^{2+}$ concentration allow hyperactivation with less interference with sperm integrity than in demembranated sperm. However, the mechanism by which hyperactivation is triggered in vivo still remains obscure.

\section{Hyperactivation and seminal plasma}

Like sperm freed from seminal plasma, boar spermatozoa can be induced to hyperactivity in a relatively narrow range of calcium concentrations if seminal plasma $(25 \%)$ is present. The range of $\mathrm{Ca}^{2+}$ concentration required for hyperactivity, however, is about 10 times higher (600 to $900 \mu \mathrm{mol} \mathrm{I}^{-1}$ ). This is likely due to the high concentrations of citrate in the seminal plasma (about $10 \mathrm{mmol} \mathrm{I}^{-1}$; Mann \& Lutwak-Mann 1981, Kamp \& Lauterwein 1995) which chelates $\mathrm{Ca}^{2+}$. As in the absence of seminal plasma, high $\mathrm{Ca}^{2+}$ concentrations $\left(>1 \mathrm{mmoll}^{-1}\right)$ caused dramatic loss of both non-hyperactive and hyperactive motility. While the percentage of hyperactive boar spermatozoa tends to be slightly more constant in the presence than in the absence of seminal plasma, the disadvantage of using seminal plasma rests in its unknown composition (e.g. decapacitation factors) and its individual variation. Therefore the calcium concentration for inducing hyperactivity had to be adjusted with respect to the seminal plasma used. To reduce individual variation, seminal plasma from several boars was pooled, divided into aliquots and stored frozen until use.

Human seminal plasma contains factors inhibiting sperm capacitation (Cross 1996). There is no definitive definition of capacitation in the literature, and several authors equate capacitation to the events leading to the 


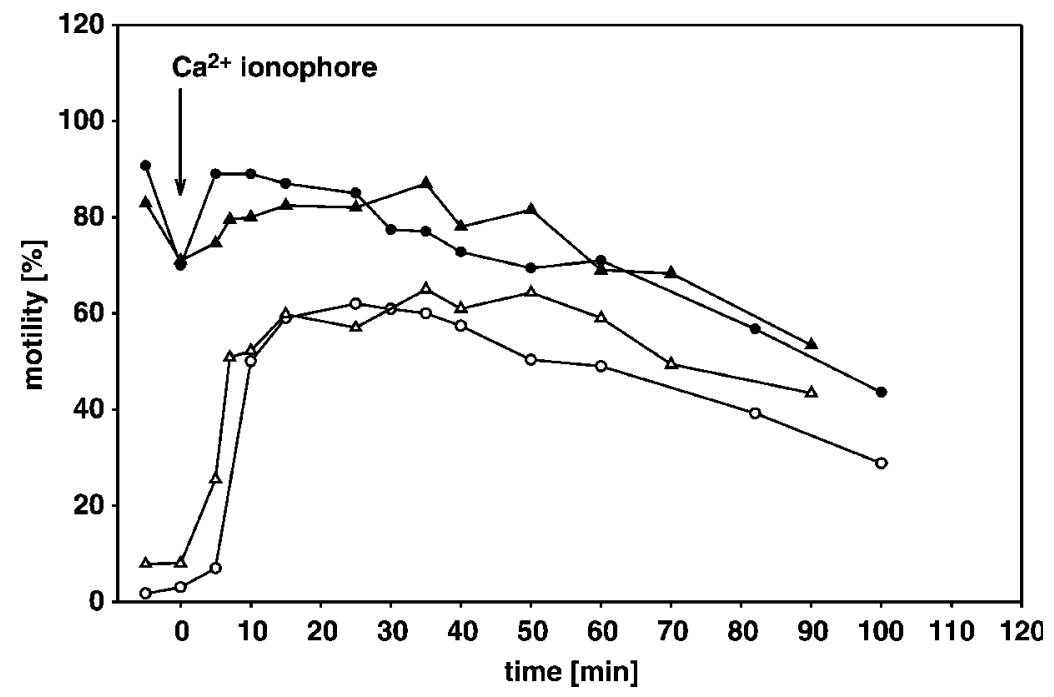

Figure 3 Time course in motility of boar spermatozoa after induction of hyperactivity. Prediluted boar semen was centrifuged $(500 \mathrm{~g}, 2 \mathrm{~min}$ ) and the spermatozoa were resuspended either in TALP-HEPES with the addition of $0.05 \mathrm{mmol} \mathrm{I}^{-1} \mathrm{Ca}^{2+}$ (circles) or in $50 \%$ TALP-HEPES, $25 \%$ BTS and $25 \%$ of seminal plasma with the addition of $0.7 \mathrm{mmoll}^{-1} \mathrm{Ca}^{2+}$ (triangles). Hyperactivation was induced by adding $5 \mathrm{mmol} \mathrm{I}^{-1} \mathrm{Ca}^{2+}$ ionophore A23187. Aliquots were analyzed by CASA after incubation for several time periods at $37^{\circ} \mathrm{C}$. The percentages of motile spermatazoa (closed symbols) and hyperactive motile spermatozoa (open symbols) were classified by multiparametric CASA.

Table 4 Quantification of hyperactive boar spermatozoa by CASA. Spermatozoa from 13 boars were evaluated by CASA. Samples were either untreated (non-hyperactivated) or hyperactivated in the absence and in the presence of $25 \%$ seminal plasma. Hyperactive spermatozoa were classified by the multiparametric definition ( $\mathrm{VCL}>97.4 \mu \mathrm{m} / \mathrm{s}$, LHD $>3.5 \mu \mathrm{m}$, LIN $<32 \%$ and WOB $<71 \%$ ). Values are given in percent means \pm S.D. $100 \%$ represents total number of spermatozoa (motile, immotile and local motile sperm). The number of boars analyzed is given in parentheses.

\begin{tabular}{lccr} 
& & \multicolumn{2}{c}{ Hyperactivated } \\
\cline { 2 - 3 } & Non-hyperactivated & Seminal plasma absent & Seminal plasma present \\
\hline Motile & $82.7 \pm 9.3(13)$ & $75.9 \pm 11.2(7)$ & $76.2 \pm 7.2(10)$ \\
Hyperactive motile & $5.1 \pm 4.3(13)$ & $48.3 \pm 6.6(7) *$ & $44.2 \pm 7.6(10) *$ \\
\hline
\end{tabular}

Significant differences $(P<0.0001)$ between non-hyperactivated and hyperactivated sperm are indicated $(*)$.

With both methods the portion of hyperactivated spermatozoa was significantly increased $(P<0.0001)$, while motility was only slightly decreased (not significant) upon hyperactivation.

acrosome reaction. However, if capacitation is more widely defined, comprising all processes of sperm maturation that make sperm ready for fertilization, and if hyperactivation is considered to be essential for fertilization, both hyperactivation and the acrosome reaction are two parts of capacitation (Yanagimachi 2003). There is some evidence that hyperactivation and acrosome reaction are induced by different extracellular factors. For example, Neill and Olds-Clarke (1987) have shown in mouse sperm that hyperactivation proceeds in the absence of BSA while acrosome-related processes are not observed (chlortetracycline assay). On the other hand, Mortimer et al. (1998) reported that hyperactivation of human spermatozoa is inhibited by $5 \%$ seminal plasma during capacitation in human tubal fluid whereas acrosome-related processes are observed (chlortetracycline assay). Intracellularly, however, both hyperactivation and the acrosome reaction seem to be triggered by the same signal $\left(\mathrm{Ca}^{2+}\right.$; Yanagimachi 1994) possibly supported by other second messengers (e.g. cAMP).

\section{Flagellar beat analysis}

Flagellar beat angle (FBA) and the flagellar curvature ratio (FCR) seem to be apt parameters for defining hyperactive spermatozoa. The average FBA values for hyperactive boar spermatozoa $\left(200^{\circ}\right)$ are similar to those of hyperactive human $\left(175^{\circ}\right)$ and lower than those for ram spermatozoa $\left(\sim 330^{\circ}\right)$. Hyperactive and non-hyperactive boar spermatozoa are well discriminated by the threshold values of FBA $\left(87^{\circ}\right)$ and FCR $(0.7)$. The FBA threshold value is the same for boar and human spermatozoa (this work and Mortimer et al. 1997), but it is considerably higher for ram spermatozoa $\left(128^{\circ}\right.$; Mortimer \& Maxwell 1999). The higher value for ram spermatozoa may be accounted for by the fact that the maximum amplitude develops closer to the head (Mortimer \& Maxwell 1999). A significant decrease in FCR from 0.93 to 0.49 upon hyperactivation has been reported for boar spermatozoa (Suarez et al. 1992), which is in good agreement with the threshold value we obtained.

\section{Multiparametric definition of hyperactive boar sperm for CASA}

CASA requires threshold values to differentiate between non-hyperactive and hyperactive spermatozoa. The values reported for human (Mortimer \& Swan 1995; for review see Mortimer 1997), ram (Mortimer \& Maxwell 1999) and rat (Cancel et al. 2000) spermatozoa are different and reflect the species-specific patterns of sperm movements. Nevertheless, the commonly used parameters to identify 
hyperactive spermatozoa are a decrease in curvilinear velocity (VCL) and linearity (LIN) as well as an increase in lateral head displacement (LHD) (cf. Mortimer 1997). For boar spermatozoa a decrease in VSL and an increase in $\mathrm{VCL}$, resulting in a reduced linearity, were reported upon hyperactivation (Suarez et al. 1992). Although there are some differences in absolute values, especially in VCL after hyperactivation, the tendency of the changes upon hyperactivation has been confirmed in the present study.

ROC analysis of selected spermatozoa suggests that the mean lateral head displacement $\left(\mathrm{LHD}_{\text {mean }}\right)$ alone is sufficient to discriminate between hyperactive and nonhyperactive spermatozoa. However, this monoparametric criterion neither considers the increased curvilinear velocity generated by deeper flagellar bending nor the decreased linearity due to the asymmetric beat pattern (Burkman 1990). Therefore, like Burkman (1990) we prefer a multiparametric definition including, at least, $\mathrm{VCL}$, LIN and LHD. The wobble (WOB) serves as an additional parameter due to its high sensitivity and specificity in ROC curve analysis.

\section{Energy metabolism of boar spermatozoa}

Hyperactivity increases the mechanical power of spermatozoa (Mortimer 1997) which is required for penetrating the zona pellucida of oocytes and consequently is vital for fertile mammalian spermatozoa (Yanagimachi 1994, Stauss et al. 1995, Mortimer 1997, Bedford 1998). The ATP supply of hyperactivity seems to require glycolysis. In the absence of glucose, hyperactivity is prevented in mouse spermatozoa even if lactate is present and oxidized in the mitochondria for ATP production (Cooper 1984, Bone et al. 2000). Lactate enables motility but cannot fuel hyperactivity. We assume that this effect is a result of the compartmentation of mitochondria in the short midpiece of the flagellum and the insufficient transport capacity for mitochondrial ATP along the flagellum (Kamp et al. 1996). The ATP supply in the long mitochondria-free part of the flagellum (principal piece) is likely met by glycolysis. Sufficient glycolytic capacity in the principal piece seems to be secured by sperm-specific glycolytic enzymes, some of which are tightly bound to the fibrous sheath surrounding the dense fibres and the axoneme along the whole principal piece (Westhoff \& Kamp 1997, Eddy et al. 2003). Because hyperactivity is essential for sperm fertility, the regulation of hyperactivity and glycolysis are very likely coordinated and should both be studied in context. Inducing and quantifying hyperactivity in boar spermatozoa as presented in this paper will allow us to follow this line of research.

\section{Acknowledgements}

We wish to thank the breeders' association GFS (Ascheberg) for providing boar semen and for their financial support. This study was also supported by the Deutsche Forschungsgemeinschaft (Ka 583/7-1,3). The authors thank Kerstin Franzen (GFS) for her cooperation, Sabine Zoernack for technical support and Gerhard Wegener for valuable comments and critical reading of the manuscript.

\section{References}

Bavister BD \& Yanagimachi R 1977 The effects of sperm extracts and energy sources on the motility and acrosome reaction of hamster spermatozoa in vitro. Biology of Reproduction 16 228-237.

Bedford JM 1998 Mammalian fertilization misread? Biology of Reproduction 59 1275-1287.

Blottner S, Bertram R \& Pitra C 1989 Species specific motility patterns of hyperactivated mammalian spermatozoa and quantitative analysis of the hyperactivation of bull spermatozoa. Andrologia 21 $204-213$.

Bone W, Jones NG, Kamp G, Yeung CH \& Cooper TG 2000 Effect of ornidazole on fertility of male rats: inhibition of a glycolysisrelated motility pattern and zona binding required for fertilization in vitro. Journal of Reproduction and Fertility 118 127-135.

Burkman LJ 1990 Hyperactivated motility of human spermatozoa during in vitro capacitation and implications for fertility. In Controls of Sperm Motility: Biological and Clinical Aspects, pp 303-330. Ed. C Gagnon. Boca Raton, FL: CRC Press.

Cancel AM, Lobdell D, Mendola P \& Perreault SD 2000 Objective evaluation of hyperactivated motility in rat spermatozoa using computer-assisted sperm analysis. Human Reproduction $\mathbf{1 5}$ $1322-1328$.

Cooper TG 1984 The onset and maintenance of hyperactivated motility of spermatozoa from the mouse. Gamete Research 9 55-74.

Cross NL 1996 Human seminal plasma prevents sperm from becoming acrosomally responsive to the agonist, progesterone: cholesterol is the major inhibitor. Biology of Reproduction 54 138-145.

Demott RP \& Suarez SS 1992 Hyperactivated sperm progress in the mouse oviduct. Biology of Reproduction 46 779-785.

Eddy EM, Toshimori K \& O'Brien DA 2003 Fibrous sheath of mammalian spermatozoa. Microscopy Research Technique 61 $103-115$.

Hamano S, Naito K, Fukuda Y \& Toyoda Y 1989 In vitro capacitation of boar ejaculated spermatozoa: effect of conditioned media prepared from preincubated sperm suspension. Gamete Research $\mathbf{2 4}$ $483-489$.

Harayama H, Miyake M, Shidara O, Iwamoto E \& Kato S 1998 Effects of calcium and bicarbonate on head-to-head agglutination in ejaculated boar spermatozoa. Reproduction, Fertility, and Development 10 445-450.

Ho HC, Granish KA \& Suarez SS 2002 Hyperactivated motility of bull sperm is triggered at the axoneme by $\mathrm{Ca}^{2+}$ and not cAMP. Developmental Biology 250 208-217.

Kamp G \& Lauterwein J 1995 Multinuclear magnetic resonance studies of boar seminal plasma. Biochimica et Biophysica Acta $1243101-109$.

Kamp G, Busselmann G \& Lauterwein J 1996 Spermatozoa: models for studying regulatory aspects of energy metabolism. Experientia 52 487-494.

Kay VJ \& Robertson L 1998 Hyperactivated motility of human spermatozoa: a review of physiological function and application in assisted reproduction. Human Reproduction Update 4 776-786.

Mann T \& Lutwak-Mann C 1981 Secretory function of the prostate, seminal vesicle, cowper's gland and other accessory organs of reproduction. In Male Reproductive Function and Semen, ch VII. Berlin: Springer-Verlag.

Mortimer ST 1997 A critical review of the physiological importance and analysis of sperm movement in mammals. Human Reproduction Update 3 403-439.

Mortimer ST 2000 CASA - practical aspects. Journal of Andrology 21 $515-524$ 
Mortimer ST \& Swan MA 1995 Variable kinematics of capacitating human spermatozoa. Human Reproduction 10 3178-3182.

Mortimer ST \& Maxwell WM 1999 Kinematic definition of ram sperm hyperactivation. Reproduction, Fertility, and Development $1125-30$.

Mortimer ST, Schevaert D, Swan MA \& Mortimer D 1997 Quantitative observations of flagellar motility of capacitating human spermatozoa. Human Reproduction 12 1006-1012.

Mortimer ST, Swan MA \& Mortimer D 1998 Effect of seminal plasma on capacitation and hyperactivation in human spermatozoa. Human Reproduction 13 2139-2146.

Neill JM \& Olds-Clarke P 1987 A computer-assisted assay for mouse sperm hyperactivation demonstrates that bicarbonate but not bovine serum albumin is required. Gamete Research 18 121-140.

Saxena N, Peterson RN, Sharif S, Saxena NK \& Russell LD 1986 Changes in the organization of surface antigens during in-vitro capacitation of boar spermatozoa as detected by monoclonal antibodies. Journal of Reproduction and Fertility 78 601-614.

Stauss CR, Votta TJ \& Suarez SS 1995 Sperm motility hyperactivation facilitates penetration of the hamster zona pellucida. Biology of Reproduction 53 1280-1285.

Suarez SS 1988 Hamster sperm motility transformation during development of hyperactivation in vitro and epididymal maturation. Gamete Research 19 51-65.

Suarez SS 2002 Formation of a reservoir of sperm in the oviduct. Reproduction in Domestic Animals 37 140-143.

Suarez SS \& Osman RA 1987 Initiation of hyperactivated flagellar bending in mouse sperm within the female reproductive tract. Biology of Reproduction 36 1191-1198.

Suarez SS \& Dai X 1992 Hyperactivation enhances mouse sperm capacity for penetrating viscoelastic media. Biology of Reproduction 46 686-691.

Suarez SS \& Ho HC 2003 Hyperactivated motility in sperm. Reproduction in Domestic Animals 38 119-124.
Suarez SS, Vincenti L \& Ceglia MW 1987 Hyperactivated motility induced in mouse sperm by calcium ionophore A23187 is reversible. Journal of Experimental Zoology 244 331-336.

Suarez S, Redfern K, Raynor P, Martin F \& Phillips DM 1991a Attachment of boar sperm to mucosal explants of oviduct in vitro: possible role in formation of a sperm reservoir. Biology of Reproduction 44 998-1004.

Suarez SS, Katz DF, Owen DH, Andrew JB \& Powell RL 1991b Evidence for the function of hyperactivated motility in sperm. Biology of Reproduction 44 375-381.

Suarez SS, Dai XB, DeMott RP, Redfern K \& Mirando MA 1992 Movement characteristics of boar sperm obtained from the oviduct or hyperactivated in vitro. Journal of Andrology 13 75-80.

Verstegen J, Iguer-Ouada M \& Onclin K 2002 Computer assisted semen analyzers in andrology research and veterinary practice. Theriogenology 57 149-179.

Westhoff D \& Kamp G 1997 Glyceraldehyde 3-phosphate dehydrogenase is bound to the fibrous sheath of mammalian spermatozoa. Journal of Cell Science 110 1821-1829.

Yanagimachi R 1969 In vitro capacitation of hamster spermatozoa by follicular fluid. Journal of Reproduction and Fertility 18 275-286.

Yanagimachi R 1994 Mammalian fertilization. In The Physiology of Reproduction, pp 189-317. Eds E Knobil \& J Neill. New York: Raven Press.

Yanagimachi R 2003 Fertilization and development initiation in orthodox and unorthodox ways: from normal fertilization to cloning. Advances in Biophysics 37 49-89.

Received 22 December 2003

First decision 11 February 2004

Revised Manuscript received 21 April 2004

Accepted 7 May 2004 